\title{
LOS LÍMITES DEL REDUCCIONISMO EN QUÍMICA
}

\section{THE LIMITS OF REDUCTIONISM IN CHEMISTRY}

\author{
Martín Labarca ${ }^{1}$
}

\begin{abstract}
Resumem: La química y la física son disciplinas científicas que abarcan un conjunto específico de teorías, cada una de ellas con sus propios conceptos y leyes. Aunque ambas disciplinas mantienen un estrecho contacto en varios campos, el problema de la relación entre ellas se manifiesta principalmente en los vínculos entre la química molecular y la mecánica cuántica: es en este caso donde generalmente se asume que las entidades químicas, cuando se las analiza en profundidad, no son más que entidades físicas muy complejas. El propósito de este trabajo es mostrar que este supuesto ampliamente compartido no sólo en la comunidad cientifica, sino también, y en general, entre los educadores en química encuentra serios obstáculos cuando se lo analiza a partir de casos concretos de estudio, tanto desde una perspectiva histórica como desde la propia práctica científica.
\end{abstract}

Palabras clave: Reduccionismo; Química molecular; Mecánica cuántica; Educación química.

Abstract: Chemistry and physics are scientific disciplines that encompass a specific set of theories, each with its own concepts and laws. Although both disciplines maintain a close contact in several fields, the problem of the relationship between them is manifested mainly in the links between molecular chemistry and quantum mechanics: it is in this case that it is generally assumed that chemical entities, when analyzed in depth, are nothing else than extremely complex physical entities. The aim of this work is to show that this assumption widely shared not only in the scientific community, but also, and in general, among chemistry educators, encounters serious obstacles when it is analyzed from concrete cases of study, both from a historical perspective as from the scientific practice itself.

Keywords: Reductionism; Molecular chemistry; Quantum mechanics; Chemistry education.

\section{Introducción}

En su caracterización más general, reduccionismo es la tesis filosófica que afirma que los ítems de un cierto ámbito de la realidad pueden obtenerse, en algún sentido, a partir de los ítems de otro ámbito. La idea de reducción, estrechamente vinculada con el ideal de unificación, atraviesa el pensamiento filosófico occidental desde sus inicios, y también se manifiesta claramente en la historia de la ciencia, en particular, de la física.

La química y la física son disciplinas científicas que abarcan un conjunto específico de teorías, cada una de ellas con sus propios conceptos y leyes. Aunque ambas disciplinas mantienen un estrecho contacto en varios campos, el problema de la relación

\footnotetext{
${ }^{1}$ Doctor Mención Ciencias Sociales y Humanas por la Universidad Nacional de Quilmes, Licenciado en Química por la Universidad Nacional de La Pampa y Profesor en Química (I.S.F.D. Nro. 45). Investigador Independiente del Consejo Nacional de Investigaciones Científicas y Técnicas (CONICET), Rosario, Santa Fe Province, Argentina. E-mail: mglabarca@gmail.com
} 
DOI: http://dx.doi.org/10.33238/ReBECEM.2019.v.3.n.1.21926

entre ellas se manifiesta principalmente en los vínculos entre la química molecular y la mecánica cuántica: es en este caso donde generalmente se asume que las entidades químicas, cuando se las analiza en profundidad, no son otra cosa que entidades físicas muy complejas. En otras palabras, la química molecular refiere a la ontología cuántica; por lo tanto, los compromisos ontológicos de la química resultan estar basados finalmente en la mecánica cuántica. Esta compleja relación entre ambas disciplinas continúa siendo un terreno muy fértil para el estudio del reduccionismo.

El propósito de este trabajo es mostrar que este supuesto ampliamente compartido no sólo en la comunidad cientifica, sino también, y en general, entre los educadores en química encuentra serios obstáculos cuando se lo analiza en profundidad a partir de casos concretos de estudio, tanto desde una perspectiva histórica como desde la propia práctica científica. Con este propósito, el trabajo se estructura de la siguiente manera: en la Sección 2 revisaremos el desarrollo histórico de la química y de la física, y la irrupción de la mecánica cuántica a comienzos del siglo XX. La distinción conceptual entre reducción epistemológica y reducción ontológica será analizada en la Sección 3. La Sección 4 está dedicada a analizar la tesis de la reducción ontológica en casos de estudio que involucran conceptos claves del mundo químico (orbital, enlace, estructura molecular, electronegatividad). Finalmente, expondremos algunas reflexiones que involucran la tesis reduccionista en la enseñanza de la química, y la importancia de la reflexión filosófica sobre la ciencia química en el aula de ciencias.

\section{El contexto del debate: la relación entre química y física}

Los historiadores de la ciencia coinciden en señalar la diferencia entre química y física en cuanto a sus orígenes históricos: mientras la física contemporánea encuentra sus raíces en el corpuscularismo mecanicista del siglo XVII, la química surge como resultado de las actividades de los alquimistas del Medioevo. A partir de estos orígenes tan disímiles, la demarcación histórica entre ambas disciplinas continúa siendo objeto de debate, debido a la búsqueda de la química de su identidad. En efecto, a lo largo de su historia esta disciplina ha atravesado una sucesión de perfiles muy variados: a fines del siglo XVII se encuentra subordinada a la física y estrechamente vinculada a la medicina y a las prácticas artesanales como la metalurgia y la perfumería. Un siglo más tarde la química es admitida como una ciencia autónoma, legítima, fundada en bases sólidas y 
DOI: http://dx.doi.org/10.33238/ReBECEM.2019.v.3.n.1.21926

fuente de útiles aplicaciones para el bien público, estatuto que logró, en gran parte, gracias a la 'revolución química' de Lavoisier. El prestigio y la autoridad, entonces, reemplazan los prejuicios que desacreditaban la imagen de la química durante el siglo XIX. En este siglo, la química ya no se considera una ciencia auxiliar de la medicina, de la farmacia o de la geología, sino como una disciplina con un fin propio, donde se alían los intereses cognitivos y comerciales (BENSAUDE-VINCENT; STENGERS, 1997). Mientras que la física tuvo como objetivo describir y explicar la realidad 'en sí misma', los principales objetivos de la química siempre implicaron la manipulación y transformación de las sustancias. Como consecuencia de ello, hasta la segunda mitad del siglo XIX la química y la física eran dos disciplinas independientes, cada una con sus propios objetivos y metodologías.

La situación comenzó a cambiar con el advenimiento de la mecánica cuántica, cuyo impactante éxito dio sustento a la idea de que la química es una rama de la física: la química supuestamente se ocupa de sistemas complejos y procesos particulares que, sin embargo, podrían ser descritos y explicados exclusivamente mediante la teoría cuántica. Ya en 1929 Paul Dirac expresaba la convicción de que, puesto que las leyes fundamentales que rigen el comportamiento de los componentes del átomo se han vuelto conocidas, hacer química significa lidiar con las ecuaciones suministradas por la física:

Las leyes subyacentes necesarias para la teoría matemática de un gran parte de la física y la totalidad de la química son así completamente conocidas, y la dificultad radica en que sólo las aplicaciones exactas de estas leyes llevan a ecuaciones que resultan demasiado complejas para ser resueltas (DIRAC, 1929, p. 714).

La afirmación de Dirac se considera habitualmente como el pronunciamiento más claro acerca de la derivabilidad de la química respecto de la física. Sin embargo, una afirmación anterior del físico Paul Langmuir en 1921 apenas se conoce, aunque expresa una posición aún más fuerte:

Creo que dentro de unos pocos años vamos a ser capaces de deducir el 90 por
ciento de todo lo que está en los libros de texto de química, deducirlo como
usted lo necesite, desde principios ordinarios simples, conociendo hechos
definidos en lo que se refiere a la estructura de los átomo (citado en SCERRI,
1994, p.162).

Esta visión adquirió rápidamente el estatus de un dogma, y fue adoptada por muchos autores que trabajan en los campos de la química, la física y la filosofía.

El problema de la relación entre química y física, generalmente conocido como 'el problema de la reducción de la química a la física', es el tópico de mayor madurez en 
DOI: http://dx.doi.org/10.33238/ReBECEM.2019.v.3.n.1.21926

la filosofía contemporánea de la química. Este problema puede plantearse de la siguiente forma: ¿Cuál es la relación entre el mundo químico y el mundo físico? ¿Es la química una disciplina científica autónoma o, por el contrario, es una mera rama de la física? ¿Existe una relación de interdependencia entre ambas disciplinas?

\section{Reducción epistemológica y reducción ontológica}

Sin duda, en la historia de la ciencia el reduccionismo ha sido una estrategia altamente exitosa para la obtención de conocimiento: la explicación de sistemas complejos a partir de sus unidades componentes fue el enfoque tradicional en el pensamiento científico desde la filosofía corpuscularista del siglo XVII. Esta perspectiva fue la que condujo a suponer la posibilidad de reducir cada ciencia especial a una más fundamental: la psicología a la biología, la biología a la química y la química a la física o, más específicamente, a las leyes de la física de las partículas elementales. El enfoque reduccionista adquiere su máxima expresión en el sueño de la llamada 'unidad de las ciencias' sostenido por el pensamiento positivista-lógico de principios del siglo XX: en la jerarquía de las ciencias naturales, la física se encontraría en la cima de la misma, mientras que la química y las demás disciplinas quedarían relegadas a una posición inferior, dado que pueden ser derivadas de las leyes fundamentales de la física.

Si bien el concepto de reducción ha sido ampliamente analizado en el ámbito de la filosofía de la ciencia, en la controversia que nos ocupa la discusión se centra en la distinción entre reducción ontológica y reducción epistemológica. La reducción ontológica supone la dependencia ontológica de las entidades y regularidades de un estrato de la realidad respecto de las entidades y regularidades de otro estrato considerado fundamental desde un punto de vista metafísico. Por lo tanto, el reduccionismo ontológico es una tesis metafísica que postula la prioridad ontológica de un cierto nivel de la realidad, al cual los restantes niveles se reducen directa o indirectamente. La reducción epistemológica se refiere, por el contrario, a la dependencia lógica entre teorías científicas: una teoría puede reducirse a otra cuando puede deducirse de aquélla. De este modo, el reduccionismo epistemológico resulta ser una tesis epistemológica según la cual la ciencia puede (o debe) ser unificada deduciendo todas las teorías científicas a partir de una única teoría privilegiada. 
DOI: http://dx.doi.org/10.33238/ReBECEM.2019.v.3.n.1.21926

Durante los últimos años, muchos filósofos de la química han desafiado este enfoque tradicional con el propósito de recuperar la autonomía de la química y, a fortiori, la legitimidad de la filosofía de la química. En algunos casos, la autonomía de la química como disciplina es defendida en términos históricos, enfatizando las diferentes tradiciones que marcaron la evolución histórica de la física y de la química ( $c f r$. VANCIK, 1999). Sin embargo, la línea de argumentación más frecuente es la que enfatiza la imposibilidad de reducción epistemológica de la química a la física. Si bien los argumentos particulares difieren entre sí, los autores concuerdan en considerar que las descripciones y los conceptos químicos no pueden derivarse de los conceptos y las leyes de la física, tal como lo supone el reduccionismo epistemológico tradicional ( $c f r$. por ejemplo, VAN BRAKEL, 2000a, Cap. 5). En este sentido, Vemulapalli y Byerly (1999) sostienen que la reducción epistemológica falla incluso en casos relativamente sencillos: la relación interteórica sólo puede establecerse introduciendo datos específicamente químicos. Desde una perspectiva similar, Scerri y McIntyre concluyen que deberíamos "renunciar a reducir epistemológicamente la química a la física" (SCERRI; McINTYRE, 1997, p. 220).

Hasta poco tiempo atrás, las discusiones acerca de la supuesta reducción de la química a la física seguían atadas a la filosofía tradicional de la ciencia del siglo XX al centrarse en cuestiones epistemológicas: la conclusión es que no todos los conceptos y leyes de la química pueden deducirse a partir de los conceptos y las leyes de la física. Sin embargo, las cuestiones ontológicas normalmente no iban más allá de aceptar incondicionalmente la reducción ontológica. Esto implicaba perder de vista una importante pregunta filosófica: ¿Por qué la química es considerada habitualmente una ciencia 'secundaria'? La respuesta a esta pregunta depende en gran medida del supuesto de la reducción ontológica: si el mundo físico reductor tiene prioridad ontológica sobre el mundo químico reducido, los conceptos químicos que no son reducibles a la mecánica cuántica refieren a entidades aparentes o secundarias dotadas de un estatus ontológico derivado; por ejemplo, la noción de forma molecular resulta ser, en palabras de WOOLLEY (1982, p. 4), sólo una "poderosa e iluminadora metáfora".

Algunos filósofos de la química han dirigido su atención a cuestiones ontológicas relacionadas con el carácter referencial de las descripciones químicas. Uno de ellos es Jaap Van Brakel (2000), quien afirma que es necesario abandonar el paradigma del espejo 
de la naturaleza, según el cual cada espejo brinda una imagen autónoma diferente del mundo (o parte de él), pero uno de los espejos -la física ideal- muestra la realidad tal como es (ontológicamente hablando): los otros espejos brindan meras apariencias sin significado cósmico. Según este autor, este paradigma debería abandonarse negando la relación asimétrica entre química y física, y afirmando que no existe descripción privilegiada. Además, van Brakel subraya el hecho de que el fracaso de la reducción epistemológica no tiene implicancia alguna en la interpretación de los conceptos químicos y su relación con lo que es 'real'. Si no hay una descripción privilegiada, tanto los conceptos químicos como los mecánico cuánticos pueden ser 'poderosas y esclarecedoras metáforas'. Sobre esta base, van Brakel concluye que deberíamos ser lo suficientemente tolerantes para dejar el mismo 'espacio ontológico' para el agua 'manifiesta', el agua en términos de la teoría termodinámica de las sustancias, la estructura molecular del agua ('construida' fuera de las mediciones espectroscópicas), las ecuaciones mecánico cuánticas 'correctas' para una molécula de agua aislada, y para los experimentos con moléculas de agua aisladas que, dependiendo de la técnica de medición, muestran aproximadamente la estructura molecular 'clásica'.

Lombardi y Labarca $(2005,2006)$ han subrayado que el fracaso de la reducción epistemológica no es lo suficientemente fuerte como para eliminar la idea de una dependencia jerárquica de la química en relación con la física. El rechazo de la posición secundaria de la química y la defensa de la legitimidad de la filosofía de la química requiere una perspectiva filosófica radicalmente diferente, que niega no sólo la reducción epistemológica sino también la reducción ontológica. En este sentido, tales autores proponen un pluralismo ontológico filosóficamente fundado para apoyar la autonomía ontológica del mundo químico y, con ello, revertir la idea tradicional de la 'superioridad' de la física en el contexto de las ciencias naturales. Mientras algunos autores se muestran escépticos acerca de los alcances de la autonomía ontológica propuesta (NEEDHAM, 2006), otros consideran que esta posición filosófica abre un camino para abordar el problema (SCERRI, 2005; McINTYRE, 2007). El debate se ha reanimado recientemente con un trabajo de Manafu (2012) donde se critica la tesis del pluralismo ontológico, y una réplica junto con nuevos argumentos en favor de la autonomía ontológica de la química (LOMBARDI, 2013, 2015). 
Al mismo tiempo, las nociones de 'emergencia' (LUISI, 2002; BISHOP, 2005) y 'superveniencia' (NEWMAN, 2008) se han propuesto para comprender la relación entre mundo químico y mundo físico. ${ }^{2}$ En este complejo escenario, se ha argumentado que, dada la enorme variedad de posibles relaciones interteóricas, la proliferación de definiciones de reducción, superveniencia, emergencia, unificación, etc., un posible camino a seguir es brindar representaciones perspicaces de la práctica de la química, vale decir, analizar casos de estudio y los nuevos debates acerca de ellos, en lugar de proseguir con disputas acerca de si la química puede ser reducida a la física, superviene de ella o ambas disciplinas pueden unificarse, y preocupaciones 'metafísicas' similares (VAN BRAKEL, 2003). Según Van Brakel (2003), la pregunta ‘¿Puede reducirse la química a la física?' carece de sentido ya que los términos ‘química' y ‘física' son demasiado vagos. Por tanto, señala el autor, necesitamos preguntas más concretas tales como ‘ ¿Se puede brindar una descripción mecánico estadística o mecánico cuántica del punto triple en el equilibrio sólido, gas, líquido?’ o bien ‘¿Podemos entender la noción de ‘sustancia química' en un discurso mecánico cuántico?'.

\section{La reducción ontológica en acción: casos de estudio}

Como fue señalado, el supuesto acerca de la reducción ontológica comenzó a ser desafiado algunos años atrás y, concretamente, a partir del estudio de casos concretos. El primero de ellos que tuvo una amplia repercusión fue el debate acerca del estatus ontológico de los orbitales atómicos. El concepto de orbital es una noción clave en la ciencia química: se utiliza para explicar enlace, estructura química y reactividad. Las discusiones acerca de su significado quedaron cómodamente confinadas al ámbito epistemológico hasta fines de la década de 1990, particularmente en el campo de la educación en química. Pero el contexto general del debate comenzó a cambiar en septiembre de 1999, cuando una noticia conmocionó a las comunidades química y física. En su portada, la prestigiosa revista Nature anunció que los orbitales atómicos $d$ de la cuprita $\left(\mathrm{Cu}_{2} \mathrm{O}\right)$ habían sido fotografiados por primera vez. Esa primera afirmación fue seguida inmediatamente por una serie de comentarios, y las imágenes obtenidas por el grupo de investigación de la Arizona State University fueron identificadas de manera

\footnotetext{
2 Para mayores detalles acerca de las nociones de reducción, emergencia y superveniencia, véase LABARCA y LASTIRI, 2017.
} 
DOI: http://dx.doi.org/10.33238/ReBECEM.2019.v.3.n.1.21926

acrítica con los orbitales atómicos, tal como se presentan en los libros de texto. La noticia fue considerada como uno de los eventos más importantes de la química en ese año, y lo mismo ocurrió en el ámbito de la física.

El informe acerca de la observación de orbitales atómicos llevó el debate directamente al terreno ontológico: ahora el problema resultaba ser la cuestión de decidir acerca de si los orbitales atómicos existen o no. En particular, en la comunidad filosófica se presentaron diferentes argumentos a la conclusión de que la visualización de orbitales es imposible por razones conceptuales. Incluso John Spence, el líder del equipo responsable del experimento, reconoció ese punto: cualquier similitud entre las imágenes reportadas y los orbitales de los libros de texto era totalmente fortuita. Aunque el debate filosófico parecía estar decidido teóricamente en favor de la no observabilidad de los orbitales, distintos experimentalistas siguieron reportando la visualización de orbitales, inclusive de orbitales moleculares (para mayores detalles, véase LABARCA; LOMBARDI, 2010).

En dos artículos publicados poco después del anuncio, Eric Scerri (2000a, 2001) enfrentó decididamente el nuevo problema ontológico y expresó su posición con claridad. Según él, los experimentos reportados como 'visualización de orbitales' fueron malinterpretados, dado que los orbitales no son observables. Y tal inobservabilidad no depende de deficiencias experimentales, sino del simple hecho de que el término 'orbital' carece de referente: los orbitales no pueden ser observados ya que, estrictamente hablando, no existen.

Este debate es una manifestación de un problema que tiene profundas consecuencias para la enseñanza de la química, ya que los docentes de la disciplina no pueden prescindir del concepto de orbital en su enseñanza. Algunos autores han señalado que esta posición choca con el supuesto según el cual la mecánica cuántica tiene la última palabra sobre el tema: sólo el concepto de función de onda es legítimo; el término 'orbital' no tiene ninguna referencia en el mundo real. En particular, el punto de vista realista sobre los orbitales adoptado por los profesores de química resulta ser incompatible con su propia posición cuando introducen la mecánica cuántica como la teoría explicativa subyacente de los fenómenos químicos. Este problema es explícitamente señalado por Scerri (2000b) cuando planteó la pregunta: ‘¿Pueden los orbitales ser reales en química pero no en física?'. 
Algunos educadores en química reaccionaron al enfoque normativista de Scerri, afirmando que la interpretación no-realista tendrá poco impacto sobre el uso de los orbitales en la enseñanza de la disciplina (EMERSON, 1999; RICHMAN, 1999). A partir de ésta y otras críticas, Scerri (2006) comenzó a mitigar su posición adoptando finalmente una posición intermedia: si bien los orbitales no pueden observarse de acuerdo con la mecánica cuántica, los profesores pueden emplearlos de manera realista pero señalando cuidadosamente sus limitaciones.

Labarca y Lombardi $(2007,2010)$ han analizado el problema del concepto de orbital a la luz de los argumentos que niegan su carácter referencial desde el punto de vista ontológico. El punto de partida fue analizar la relación entre mecánica cuántica y química molecular. Mientras que en mecánica cuántica 'orbital' es un término que no refiere, en química molecular los orbitales existen como regiones espaciales que explican la forma de las moléculas locales e individuales. El argumento para rechazar la existencia de orbitales, señalan los autores, se basa en una premisa fundamental: la identificación entre 'orbital' y 'función de onda'. Los autores ponen en cuestión tal identificación preguntándose entonces si éste es el concepto de orbital utilizado en química para hablar de las formas de los orbitales o para explicar el enlace químico.

El problema del estatus ontológico de la estructura molecular concita también la atención de químicos y filósofos de la química. La teoría molecular clásica concibe la estructura molecular como un arreglo tridimensional de átomos, unidos entre sí mediante enlaces químicos. El conocimiento de la naturaleza de los enlaces así como de la geometría espacial que presenta la molécula es esencial a la hora de predecir su reactividad química. Sin embargo, dicho concepto parece perder su referente en una ontología como la descripta por la teoría cuántica, dada la indeterminación de la posición y del momento de los componentes moleculares. Pero, al mismo tiempo, el concepto de estructura molecular ocupa un lugar central en las explicaciones químicas.

La estrategia tradicional para vincular las descripciones químicas y cuánticas de las moléculas suele apelar a la aproximación de Born-Oppenheimer propuesta en 1927. Dicha aproximación es utilizada para sistemas moleculares de N-partículas, y cuya ecuación, como es sabido, no tiene solución analítica. La separación de los movimientos nucleares y electrónicos depende de asumir los núcleos como partículas clásicas en reposo y en una posición definida. Esta aproximación puede pensarse teniendo en cuenta que la 
DOI: http://dx.doi.org/10.33238/ReBECEM.2019.v.3.n.1.21926

masa nuclear $M$ es mucho mayor que la masa electrónica $m$, de manera que dicha relación es aproximadamente $m / M \rightarrow 0$. Tal límite es habitualmente el supuesto que conduce al concepto de estructura molecular. Pero las masas nunca son infinitas: esto es lo que hace a la aproximación de Born-Oppenheimer una aproximación y no un método preciso. Los límites de esta estrategia reductiva han sido ampliamente debatidos en la bibliografía sobre el tema ( $c f r$. por ej. AMANN, 1992; PRIMAS, 1998; LOMBARDI; CASTAGNINO, 2010).

En este contexto, mientras algunos autores adoptan una posición explícitamente reduccionista y abogan por reconstruir el concepto de estructura molecular en el marco de la teoría cuántica de átomos en moléculas (HETTEMA, 2012), otros apelan al concepto de emergencia para entender dicha noción (HENDRY, 2010). Por su parte, Sutcliffe y Woolley $(2011,2012)$ afirman que la imposibilidad de derivar la estructura molecular a partir de la mecánica cuántica no es concebida como un obstáculo conceptual, sino como la consecuencia de nuestro conocimiento parcial de los sistemas moleculares en el marco de la mecánica cuántica. Otros autores proponen compatibilizar ambos marcos teóricos (química molecular y mecánica cuántica) desde una perspectiva pluralista de análisis sincrónico, según el cual el término 'estructura molecular' encuentra su referente en una ontología química molecular y carece del mismo en una ontología de partículas (FORTIN; LABARCA; LOMBARDI, 2018).

Otro caso de estudio concierne con la naturaleza del enlace químico covalente. Como es sabido, la noción de enlace constituye uno de los conceptos centrales de la ciencia química. Más aún, gran parte de la química moderna se ocupa de las propiedades y la dinámica de los enlaces químicos. Las teorías actuales sobre el tema surgen de los trabajos de Irving Langmuir, Walther Kossel y, muy especialmente, de Gilbert Newton Lewis en 1916. Con una notable intuición, Lewis desarrolló su teoría varios años antes del surgimiento de la mecánica cuántica y del concepto de orbital. Con el advenimiento de la mecánica cuántica, dos 'teorías' químico cuánticas brindaron una descripción del enlace covalente en térmicos formales: la teoría del enlace de valencia y la teoría de los orbitales moleculares.

La teoría clásica del enlace de valencia (EV) tiene su origen en el artículo que Walter Heitler y Fritz London publicaron en 1927. Este trabajo fue la primera aplicación de la mecánica cuántica a un sistema molecular, la molécula de hidrógeno $\mathrm{H}_{2}$, con el 
DOI: http://dx.doi.org/10.33238/ReBECEM.2019.v.3.n.1.21926

propósito de describir el enlace covalente. ${ }^{3}$ La generalización a moléculas poliatómicas se debió a los trabajos de John C. Slater, del premio Nobel Linus Pauling, de Henry Eyring y Michael Polanyi, y de George Willard Wheland. La EV es la primera descripción mecánico-cuántica del enlace covalente en términos de orbitales atómicos. Esta teoría está basada en ideas estructurales previas y, en particular, en la teoría electrónica de Lewis, proporcionando una forma de calcular los valores numéricos de los ángulos y las longitudes de enlace.

Por su parte, la teoría de los orbitales moleculares (OM) fue introducida por el premio Nobel Robert Mulliken en 1928 y Friedrich Hund, con importantes contribuciones de Sir John Lennard-Jones, Erich Hückel y Charles Coulson. Esta teoría surge como una alternativa superadora de los límites que presentaban la teoría electrónica de Lewis y la teoría de repulsión de los pares de electrones del nivel de valencia (VSEPR, por sus siglas en inglés), y era más fácil de utilizar en los cálculos que la EV.

Si bien ambos enfoques emergieron casi simultáneamente y se transformaron en escuelas rivales en su tiempo, es importante resaltar que en la actualidad conviven en la comunidad científica los tres marcos teóricos reseñados. Tal como señalan varios autores, no sólo la EV y la OM desempeñan papeles complementarios (HIBERTY; SHAIK, 2008), sino que ellas no han eclipsado, además, a la teoría de Lewis, una teoría química supuestamente 'derivada' o 'secundaria'. La utilización de las mismas dependerá de los factores considerados relevantes a tal propósito: facilidad de implementación computacional, facilidad de uso en la enseñanza, facilidad en la visualización, etc. Es aquí donde acudir a la noción de 'modelo' ayuda a disipar las perplejidades que derivan de la coexistencia de descripciones y explicaciones diferentes, e incluso incompatibles, de un mismo fenómeno (ADÚRIZ-BRAVO; LABARCA; LOMBARDI, 2014).

Un último ejemplo a brindar es el concepto de electronegatividad, una noción relevante en química a partir de la segunda mitad del siglo XX, para explicar el comportamiento de las sustancias cuando encuentran otras sustancias. Si bien los químicos no acuerdan acerca de qué es la electronegatividad, en su versión tradicional expresa la habilidad, capacidad o propiedad de las especies químicas para atraer electrones en enlaces compartidos. En 1932 Linus Pauling proponía la primera definición

${ }^{3}$ Históricamente, se considera que la descripción del enlace químico utilizando por primera vez una teoría física brindó las bases para afirmar que la química puede ser reducida a la física cuántica (HARRIS, 2008). 
DOI: http://dx.doi.org/10.33238/ReBECEM.2019.v.3.n.1.21926

del concepto y cuantificó la electronegatividad sobre la base de una propiedad termoquímica como la energía de disociación de enlaces en sustancias de tipo $A_{n} B_{m}$. El modelo de Pauling se actualizó posteriormente con mediciones más precisas, y ha servido como una guía para otros modelos de electronegatividad.

Dado que la electronegatividad no es un observable físico, no se puede determinar experimentalmente. En consecuencia, se han publicado docenas de modelos basadas en una amplia variedad de propiedades químicas y físicas. En la actualidad, es posible identificar al menos tres enfoques de cuantificación diferentes: el termoquímico, el espectroscópico y el electrostático (RUTHENBERG; MARTÍNEZ GONZÁLEZ, 2017).

Murphy et al. (2000) establecieron un conjunto de reglas que una escala de electronegatividad aceptable debería cumplir. Uno de ellas afirma que la electronegatividad debe tener una definición mecánico-cuántica viable, es decir, compatible con los conceptos cuánticos elementales. Pese a ello, la definición de electronegatividad de Pauling en un dominio termoquímico junto con la definición de Mulliken, en un dominio espectroscópico, continúan siendo, en general, las primeras aproximaciones al tema en los textos de enseñanza de química. La coexistencia de modelos incompatibles de electronegatividad es motivo de análisis filosófico en la actualidad (ACCORINTI, 2019).

En resumen, podríamos tener buenas razones para admitir la reducción ontológica de los ítems 'secundarios' si el abandono o la modificación de la teoría o modelo supuestamente fundamental implicara cambios profundos en la teoría o modelo que describe tales ítems: en tal caso podría sostenerse que las características del dominio 'secundario' dependen o son de algún modo el resultado de las características de la ontología 'fundamental'. Pero si la suerte de la teoría o modelo 'secundario' o 'fenomenológico' es inmune a la suerte que corra la teoría o modelo 'fundamental', no parece haber buenos motivos filosóficos para suponer que el dominio descrito por la primera se reduce ontológicamente al dominio descrito por la segunda.

\section{Conclusiones}

Hemos reseñado los debates actuales en el campo de la filosofía de la química respecto de algunos conceptos relevantes de la disciplina y como la tesis de la reducción ontológica impregna fuertemente tales controversias. Contrariamente al supuesto 
habitualmente aceptado, ni la historia ni la práctica científica de la química favorecen el enfoque monista; por el contrario, la perspectiva pluralista (la utilización de distintas teorías y modelos para explicar el mismo fenómeno) se constituye en el enfoque para obtener conocimiento. Pero aun si aceptaramos la tesis reduccionista, conceptos como orbital, enlace, estructura molecular y electronegatividad, entre otros, carecerían estrictamente de referente en un dominio químico. ¿Cuál sería la consecuencia para la enseñanza de la disciplina?

Parece bastante claro que esta situación paradójica tendría consecuencias negativas para una profunda comprensión de la disciplina por las siguientes razones: a) los estudiantes se enfrentan a la alternativa de vivir en una especie de 'esquizofrenia conceptual' o aceptar que la química describe fenómenos meramente aparentes o 'metafóricos'; y b) si la definición de los conceptos reseñados sólo radica en el campo de la física -en particular, a partir de los dictados de la teoría cuántica-, los estudiantes que deciden estudiar química deberían acudir a los libros de texto de otra disciplina. Esta situación parece ser también inadecuada desde el punto de vista educativo. Y en este sentido, algunos autores observan que la enseñanza de la química en el nivel medio, y aún en el nivel universitario, no sólo no es deseable desde una perspectiva estrictamente formal de la mecánica cuántica sino que es impracticable (NAHUM et al. 2008).

Uno de los principales tópicos en el campo de la educación en ciencias desde mediados de la década de 1990 es la argumentación en clase (ERDURAN; JIMÉNEZALEIXANDRE, 2007). Los debates vistos aquí muestran que la filosofía de la química se constituye en una excelente herramienta pedagógica para favorecer los procesos de argumentación en el aula de química. Ello redundará en un conocimiento más profundo de la naturaleza de una fascinante ciencia natural como la química.

\section{Agradecimientos}

Este trabajo fue posible gracias al apoyo de la Universidad de Buenos Aires (subsidio UBACyT 20020170200026BA) de Argentina.

\section{Referencias}

ACCORINTI, H. Incompatible models in chemistry: the case of electronegativity. Foundations of Chemistry, Dordrecht, v. 21, n. 1, p. 71-81, 2019. 
ADÚRIZ-BRAVO, A.; LABARCA, M.; LOMBARDI, O. Una noción de modelo útil para la formación del profesorado de química. En: MERINO, C.; ADÚRIZ-BRAVO, A. y ARELLANO, M. (eds.). Avances en Didáctica de la Química: Modelos y Lenguajes. Valparaíso: Ediciones Universitarias de Valparaíso, 2014. p. 37-49.

AMANN, A. Must a molecule have a shape? South African Journal of Chemistry, Pretoria, v. 45, p. 29-38, 1992.

BENSAUDE-VINCENT, B.; STENGERS, I. Historia de la Química. Madrid: AddisonWesley and Universidad Autónoma de Madrid, 1997.

BISHOP, R. Patching physics and chemistry together. Philosophy of Science, Baltimore, v. 72, p.710-722, 2005.

DIRAC, P. A. M. Quantum mechanics of many-electron systems. Proceedings of the Royal Society, Edinburgh, v. 123, n. 792, p.714-733, aprl. 1929.

EMERSON, K. The quantum mechanical explanation of the periodic system. Journal of Chemical Education, Easton, v. 76, n.9, p.1189, 1999.

ERDURAN, S.; JIMÉNEZ-ALEIXANDRE, M. P. (eds.). Argumentation in Science Education. Perspectives from Classroom Based Research, Netherlands: Springer, 2007.

FORTIN, S., LABARCA, M.; LOMBARDI, O. On the ontological status of molecular structure: is it possible to reconcile molecular chemistry with quantum mechanics?

Foundations of Chemistry, Dordrecht, enviado para su publicación, 2018.

HARRIS, M. L. Chemical reductionism revisited: Lewis, Pauling and physico-chemical nature of chemical bond. Studies in History and Philosophy of Science, Elmsford, v. 39, p. 78-90, 2008.

HENDRY, R. F. Ontological reduction and molecular structure. Studies in History and Philosophy of Modern Physics, v. 41, p. 183-191, 2010.

HETTEMA, H. Reducing Chemistry to Physics: Limits, Models, Consequences. Groningen: University of Groningen, 2012.

HIBERTY, P. C.; SHAIK, S. A Chemist Guide to Valence Bond Theory. Hoboken, New Jersey: John Wiley \& Sons, Inc., 2008.

LABARCA, M.; LASTIRI, M. Relaciones interteóricas. En: VANNEY, C. E.; SILVA, I.; FRANCK, J. F. Diccionario Interdisciplinar Austral, 2017. Disponible en: <http://dia.austral.edu.ar/Relaciones_interteóricas>. Acceso en: 20 ene. 2019.

LABARCA, M.; LOMBARDI, O. Why orbitals do not exist? Foundations of Chemistry, Dordrecht, v. 12, p. 149-57, 2010.

LOMBARDI, O. ¿Acerca de qué nos habla la química? Nuevos argumentos en favor de la autonomía ontológica del mundo químico. Revista Colombiana de Filosofía de la Ciencia, Bogotá, v. 13, n. 26, p.105-144, 2013. 
DOI: http://dx.doi.org/10.33238/ReBECEM.2019.v.3.n.1.21926

LOMBARDI, O. The ontological autonomy of the chemical world: facing the criticisms. En: SCERRI, E.; McINTYRE, L. (eds.), Philosophy of Chemistry: Growth of a New Discipline (Boston Studies in the Philosophy and History of Science). Dordrecht: Springer, 2015. p. 23-38.

LOMBARDI, O.; CASTAGNINO, M. Matters are not so clear on the physical side.

Foundations of Chemistry, Dordrecht, v.12, p. 159-166, 2010.

LOMBARDI, O.; LABARCA, M. The ontological autonomy of the chemical world. Foundations of Chemistry, Dordrecht, v.7, n. 2, p. 125-148, 2005.

LOMBARDI, O.; LABARCA, M. The ontological autonomy of the chemical world: a response to Needham. Foundations of Chemistry, Dordrecht, v. 8, n. 1, p. 81-92, 2006.

LOMBARDI, O.; LABARCA, M. The philosophy of chemistry as a new resource for chemistry education. Journal of Chemical Education, Easton, v. 84, n. 1, p. 187-192, 2007.

LUISI, P. L. Emergence in chemistry: Chemistry as the embodiment of emergence.

Foundations of Chemistry, Dordrecht, v. 4, p. 183-200, 2002.

MANAFU, A. Internal realism and the problem of ontological autonomy: A critical note on Lombardi and Labarca. Foundations of Chemistry, Dordrecht, v. 15, p. 225-228, 2012.

McINTYRE, L. The philosophy of chemistry: ten years later. Synthese, Dordrecht, v. 155, n. 3, p. 291-292, 2007.

MURPHY, L.; MEEK, T.; ALLRED, A.; ALLEN, L. Evaluation and test of Pauling's electronegativity scale. Journal of Physical Chemistry A, Washington, v. 104, p. 5867-5871, 2000.

NAHUM, T. L.; MAMLOK-NAHMAN, R.; HOFSTEIN, A.; KRONIK, L. A New "BottomUp" Framework for Teaching Chemical Bonding. Journal of Chemical Education, Easton, v. 85 , n. 12, p. 1680-1685, 2008.

NEEDHAM, P. Ontological reduction: comment on Lombardi and Labarca. Foundations of Chemistry, Dordrecht, v. 8, n. 1, p. 73-80, 2006.

NEWMAN, M. Chemical supervinience. Foundations of Chemistry, Dordrecht, v. 10, p. 49$62,2008$.

PRIMAS, H. Emergence in exact natural sciences. Acta Polytechnica Scandinavica, Stockholm, v. 91, p. 83-98, 1998.

RICHMAN, R. In defense of quantum numbers. Journal of Chemical Education, Easton, v. 76, n. 5, p. 608, 1999.

RUTHENBERG, K.; MARTÍNEZ GONZÁLEZ, J. C. Electronegativity and its multiple faces: persistence and measurement. Foundations of Chemistry, Dordrecht, v.19, p. 61-75, 2017.

SCERRI, E. R. Editorial 20. Foundations of Chemistry, Dordrecht, v.7, n. 2, p. 119-123, 2005.

SCERRI, E. R. Has chemistry been at least approximately reduced to quantum mechanics?

PSA: Proceedings of the Biennial Meeting of the Philosophy of Science Association, v. 1, n. 1, p. 160-170, 1994. 
DOI: http://dx.doi.org/10.33238/ReBECEM.2019.v.3.n.1.21926

SCERRI, E. R. Have orbitals really been observed? Journal of Chemical Education, Easton, v. 77, n. 11, p. 1492-1494, 2000a.

SCERRI, E. R. Philosophy of chemistry: a new interdisciplinary field? Journal of Chemical Education, Easton, v. 77, n. 20, p. 522-525, 2000 b.

SCERRI, E. R. The recently claimed observation of atomic orbitals and some related philosophical issues. Philosophy of Science 68 (Proceedings), p. S76-S78, 2001.

SCERRI, E. R.; McINTYRE, L. The case for the philosophy of chemistry. Synthese, Dordrecht, v. 111, n. 3, p. 213-232, 1997.

SUTCLIFFE, B.; WOOLLEY, R. G. A comment on Editorial 37. Foundations of Chemistry, Dordrecht, v. 13, p. 93-95, 2011.

SUTCLIFFE, B.; WOOLLEY, R. G. Atoms and molecules in classical chemistry and quantum mechanics. In: WOODY, A.; NEEDHAM P.; HENDRY, R. F. (eds.). Handbook of

Philosophy of Science. v. 6. Philosophy of Chemistry, Oxford: Elsevier, 2012. p. 387-426.

VAN BRAKEL, J. Philosophy of Chemistry. Between the Manifest and the Scientific Image. Leuven: Leuven University Press, 2000.

VAN BRAKEL, J. The Ignis Fatuus of reduction and unification: back to the rough ground. In: EARLEY, J. (ed.). Chemical Explanation: Characteristics, Development, Autonomy. New York: New York Academy of Sciences, 2003. p. 30-43.

VANCIK, H. Opus magnum: an outline for the philosophy of chemistry. Foundations of Chemistry, Dordrecht, v. 1, n. 3, p. 242-256, 1999.

VEMULAPALLI, G. K.; BYERLY, H. Remnants of reductionism. Foundations of Chemistry, Dordrecht, v. 1, n. 1, p. 17-41, 1999.

WOOLLEY, R. G. Natural optical activity and the molecular hypothesis. Structure and Bonding, Berlin, v. 52, n. 1, p. 1-35, 1982.

Convidado em: 01 de fevereiro de 2019.

Submetido em: 14 de março de 2019.

Revisado em: 25 de abril de 2019. 